\title{
Inhibition Activity of Three Kind of Antibiotics for Carbon Steel Corrosion in Acetic Acid Environment
}

\author{
Ziwen Wang ${ }^{1}$, Wei Bai ${ }^{1, *}$, Cunying $\mathrm{Xu}^{2}$, Yulu Shi ${ }^{1}$, You $\mathrm{Wu}^{1}$, Junming Guo ${ }^{1}$ and Lili Feng ${ }^{1}$ \\ ${ }^{1}$ Key Laboratory of Resource Clean Conversions in Ethnic Regions, Yunnan Minzu University, \\ Kunming, 650500, PR China. \\ ${ }^{2}$ Faculty of Metallurgical and Energy Engineering, Kunming University of Science and Technolo-gy, \\ Kunming, 650500, PR China. \\ *E-mail: bw369852147@qq.com
}

doi: $10.20964 / 2017.11 .79$

Received: 17 August 2017 / Accepted: 22 September 2017 / Published: 12 October 2017

\begin{abstract}
The inhibition mechanism of carbon steel in acetic acid medium in the presence of amoxicillin, cefaclor or cephradine has been studied with the application of weight loss measurements, potentiodynamic polarization curves, EIS, and SEM. In order to investigate the corrosion mechanism more deeply, we characterized the frontier molecular orbital energy $\left(\mathrm{E}_{\mathrm{HOMO}}, \mathrm{E}_{\mathrm{LUMO}}\right)$, Mulliken atomic population analysis and charge density difference by using DFT study. In the case where the spatial structure is similar in resistance, the effects of cefradine on cefradine and cefaclor were as follows: the inhibitory effect of cefradine in the three drugs was the best and its inhibition rate was $87.7 \%$.The maximum of cefaclor and amoxicillin is only $63.5 \%$ and $52.1 \%$ at the same concentration. Electrochemical test indicated that cephradine and cefaclor behaved as cathode type inhibitors and amoxicillin behaved as mixed type inhibitor. These results of experimental and theoretical calculations were consistent and they showed that cephradine was the most effective inhibitor.
\end{abstract}

Keywords: Antibiotic, Carbon steel, Corrosion inhibition, Acetic acid, Theoretical calculation

\section{$\underline{\text { FULL TEXT }}$}

(C) 2017 The Authors. Published by ESG (www.electrochemsci.org). This article is an open access article distributed under the terms and conditions of the Creative Commons Attribution license (http://creativecommons.org/licenses/by/4.0/). 